Research Paper

\title{
Epidemiological, Clinical, Radiological Characteristics and Outcomes of Medical Staff with COVID-19 in Wuhan, China: Analysis of 101 Cases
}

\author{
Jie Liu ${ }^{1,2^{*}}$, Liu Ouyang $3^{3 *}$, Dan Yang4, Xiaoyu Han ${ }^{1,2}$, Yukun Cao ${ }^{1,2}$, Osamah Alwalid ${ }^{1,2}$, Hanping Wu ${ }^{5}$, \\ Heshui Shi ${ }^{1,2}$, Fan Yang ${ }^{1,2}$, Chuansheng Zheng ${ }^{1,2}$ \\ 1. Department of Radiology, Union Hospital, Tongji Medical College, Huazhong University of Science and Technology, Wuhan 430022, China. \\ 2. Hubei Province Key Laboratory of Molecular Imaging, Wuhan 430022, China. \\ 3. Department of Orthopaedics, Union Hospital, Tongji Medical College, Huazhong University of Science and Technology, Wuhan 430022, China. \\ 4. Department of Respiratory and Critical Care Medicine, Union Hospital, Tongji Medical College, Huazhong University of Science and Technology, Wuhan \\ 430022, China. \\ 5. Department of Radiology, Michigan Medicine, University of Michigan, Michigan, The United States of America. \\ * These authors contributed equally in this work.
}

$\triangle$ Corresponding author: Fan Yang, Department of Radiology, Union Hospital, Tongji Medical College, Huazhong University of Science and Technology, Wuhan 430022, China (Email: fyang@hust.edu.cn); Chuansheng Zheng, Department of Radiology, Union Hospital, Tongji Medical College, Huazhong University of Science and Technology, Wuhan 430022, China (Email: hqzcsxh@sina.com).

(c) The author(s). This is an open access article distributed under the terms of the Creative Commons Attribution License (https://creativecommons.org/licenses/by/4.0/). See http:/ /ivyspring.com/terms for full terms and conditions.

Received: 2020.10.09; Accepted: 2021.01.06; Published: 2021.01.29

\begin{abstract}
Objectives: As of 11 Feb 2020, a total of 1,716 medical staff infected with laboratory-confirmed the severe acute respiratory syndrome coronavirus 2 (SARS-Cov-2) in China had been reported. The predominant cause of the infection among medical staff remains unclear. We sought to explore the epidemiological, clinical characteristics and prognosis of infected medical staff.

Methods: Medical staff who infected with SARS-Cov-2 and admitted to Union Hospital, Wuhan between 16 Jan to $25 \mathrm{Feb}, 2020$ were included in this single-centered, retrospective study. Data were compared by occupation and analyzed with the Kaplan-Meier and Cox regression methods.

Results: A total of 101 medical staff ( 32 males and 69 females; median age: 33 ) were included in this study and $74.3 \%$ were nurses. A small proportion of the cohort had contact with specimens (3\%) as well as patients infected with SARS-Cov-2 in fever clinics (15\%) and isolation wards (3\%). $80 \%$ of medical staff showed abnormal IL-6 levels and 33\% had lymphocytopenia. Chest CT mainly manifested as bilateral (62\%), septal/subpleural (77\%) and ground-glass opacities (48\%). The major differences between doctors and nurses manifested in laboratory indicators. As of the last observed date, no patient was transferred to intensive care unit or died. Fever $(\mathrm{HR}=0.57 ; 95 \% \mathrm{Cl} 0.36-0.90)$ and IL-6 levels greater than $2.9 \mathrm{pg} / \mathrm{ml}(\mathrm{HR}=0.50 ; 95 \% \mathrm{Cl} 0.30-0.86)$ were unfavorable factors for discharge.

Conclusions: Our findings suggested that the infection of medical staff mainly occurred at the early stages of SARS-CoV-2 epidemic in Wuhan, and only a small proportion of infection had an exact mode. Meanwhile, medical staff infected with COVID-19 have relatively milder symptoms and favorable clinical course than ordinary patients, which may be partly due to their medical expertise, younger age and less underlying diseases. The potential risk factors of fever and IL-6 levels greater than $2.9 \mathrm{pg} / \mathrm{ml}$ could help to identify medical staff with poor prognosis at an early stage.
\end{abstract}

Key words: COVID-19; Medical staff; Infectious disease; Epidemiology; Clinical features; Computerized tomography.

\section{Introduction}

In Dec 2019, a group of novel atypical pneumonia patients with uncertain etiology but mostly linked to the Huanan Seafood Wholesale
Market emerged in Wuhan, China [1]. A later confirmed pathogen of this previously unknown pneumonia was described as a novel coronavirus, 
currently named as severe acute respiratory syndrome coronavirus 2 (SARS-Cov-2), was ascertained by unbiased sequencing analysis of lower respiratory tract samples from early cases on 7 Jan 2020, following which the protocol of real-time reverse-transcriptase polymerase chain reaction (RT-PCR) assay for this novel coronavirus had also been developed [2,3]. By 5 October 2020, coronavirus disease 2019 (COVID-19) due to the SARS-Cov-2 has caused 34,804,348 cases laboratory confirmed cases and 1,030,738 deaths among them globally [4]. Sufficient evidence indicated that the COVID-19 clustered within close-contact human groups, such as family and hospital settings [5-7].

Information pointing to the epidemiology and clinical features of general confirmed cases has been accumulating $[1,8,9]$. Meanwhile, a finding from a national wide descriptive report drew a huge amount of attention, which declared that the total number of confirmed novel coronavirus-infected medical staff was as high 1,716 as of 11 February 2020, with a peak incidence occurring on Jan 28, 2020 [10]. Among them, $63 \%$ (1080) were in Wuhan [10]. A cluster of 14 medical staff infected with COVID-19 from department of neurosurgery in Wuhan Union Hospital were initially reported [11]. The previous studies enrolling 14, 80 and 54 hospitalized frontline medical workers infected with COVID-19 respectively, provided an insight into epidemiological and clinical characteristics of these patients [11-13]. Nonetheless, information regarding the clinical outcomes and potential risk factors of medical staff confirmed with COVID-19 remains to be investigated.

At present, there are increased attentions paid to protecting medical staff from infection, and medical staff are regarded as every country's most valuable resource to fight against the COVID-19 outbreak [14]. People paid tribute to healthcare workers for their efforts during the outbreak of COVID-19 [15]. Based on a group of medical staff confirmed with COVID-19 who were admitted to Union Hospital, Wuhan, this retrospective study aimed to reveal some epidemiological and clinical findings, and identify potential risk factors of extended hospitalization. We hope the findings will provide an insight into the prevention and treatment of this novel coronavirus for the global community.

\section{Patients and Methods}

This retrospective study was approved by the Ethics of Committees of Union Hospital, Tongji Medical College, Huazhong University of Science and Technology. Written informed consent was waived due to the rapid emergence of this infectious disease.

\section{Study design and participants}

This is a single-centered, retrospective study on a group of SARS-CoV-2 infected medical staff at Wuhan Union Hospital, one of the hospitals treating patients with COVID-19 at the earliest time. Diagnosis of cases with SARS-Cov-2 infection conforms to the WHO interim guidance [16]. Details regarding laboratory confirmation protocol for SARS-CoV-2 were described by previous studies [1, 9]. Throat-swab specimens were screened for SARS-CoV-2 and other respiratory viruses (influenza, respiratory syncytial virus, etc.) by real-time RT-PCR assays. This study only considered medical staff that are in service. A total 101 medical staff, which were confirmed by SARS-CoV-2 real-time RT-PCR test on respiratory secretions collected by throat swab and undergone serial chest CT scans following their admission to isolation wards of Union Hospital between 16 Jan and $25 \mathrm{Feb}, 2020$ were included.

\section{Data collection}

The epidemiological data, medical and nursing records, laboratory examinations, chest computed tomography (CT) of all patients were reviewed and abstracted with concerted efforts of experienced clinicians. Data were collected at the time of symptoms onset, presentation for medical advice and in-patient admission. The clinicians who had experience of treating patients with confirmed SARS-Cov-2 infection reviewed and collected the medical records of patients, and preliminarily collated the data. The clinical data were extracted through a standardized form for case report as previously described [17]. Epidemiological data, including exposure histories before symptoms onset (whether there is a history of exposure to the Huanan Seafood Wholesale Market, or wildlife), and close contact with laboratory-confirmed or suspected cases of COVID-19 in work environment (fever clinics, or isolation wards) and sample collection sites (with pharyngeal swab, blood, sputum specimens, etc.), or close contact with family members with COVID-19 were collected. Also, information about preventive medication among medical staff was collected.

We have also collected the data on demographics, clinical manifestations, laboratory examinations and radiological studies. These included age, sex, occupation (doctor, or nurse), body mass index (BMI $\geq 24$, or $\left.<24 \mathrm{~kg} / \mathrm{m}^{2}\right)$, current smoking status (yes, or no), disease severity (non-severe, or severe), date of symptom onset, diagnosis and hospital admission, symptoms before hospital admission (fever, cough, fatigue, sore throat, myalgia, sputum production, difficulty breathing or chest tightness, chill, loss of appetite, diarrhea, and chest 
pain), coexisting conditions (e.g. hypertension, diabetes, etc.), laboratory testing indicators on admission (leucocyte count, lymphocyte count, platelet count, D-dimer, creatinine, creatine kinase, lactose dehydrogenase, alanine aminotransferase, aspartate aminotransferase, hemoglobin, ferritin, C-reactive protein, Amyloid A, total bilirubin, procalcitonin, erythrocyte sedimentation rate, interleukin-6 (IL-6) and lymphocyte subsets, etc.), radiologic assessments of chest CT (lung involvement, lung lobe involvement, predominant CT changes, predominant distribution of opacities, etc.), treatment measures (antibiotics agents, antiviral agents, traditional Chinese medicine, immune globulin, thymosin, corticosteroids and oxygen therapy), and complications (e.g. pneumonia, acute respiratory distress syndrome, acute cardiac injury, acute kidney injury, shock, etc.). All CT images were analyzed by two radiologists (J.L. and F.Y., who had 5 and 21 years of experience in thoracic radiology, respectively) utilizing the institutional digital database system without access to clinical and laboratory findings. Images were reviewed independently, and final decisions were reached by discussion and consensus. We estimated the time interval from symptom onset to diagnosis and admission with maximum information available - that is, all the exact date of initial symptoms provided by the patients. Then the aggregated data was sent to data analysis group. Prior to statistical analysis, the aggregated data were cross checked by group members to guarantee the correctness and completeness of data.

\section{Outcomes}

The clinical outcomes and prognosis were continuously observed up to Mar 20, 2020. The primary end point was discharge, needed to meet the following three conditions [18]: (1) body temperature return to normal for more than 3 days and respiratory symptoms improvement; (2) improvement of lung involvement demonstrated by chest CT; (3) two consecutive negative RT-RCR tests, with sampling interval of more than 1 day. Secondary outcomes consisted of hospital discharge rate.

\section{Statistical analysis}

This study devoted to report epidemiological, clinical characteristics and prognosis of medical staff confirmed with COVID-19. We estimated the distributions of durations from symptoms onset to diagnosis, symptoms onset to admission, and diagnosis to admission, respectively. Kaplan-Meier method was applied to estimate the change in hospital discharge rate. The proportional hazard Cox regression model was utilized to ascertain potential factors associated with discharge. Univariate models with a single variable once at a time were first fitted. The statistically significant risk factors as well as age and sex were, then, would be considered and selected into a final multivariate Cox regression model. The hazards ratios (HRs) along with the 95\% confidence intervals (95\% CIs) were calculated.

Statistical tests were two-sided with significance set at $a$ less than 0.05 . We performed all data analyses by $R$ software version 3.6.2 ( $R$ Foundation for Statistical Computing).

\section{Results}

\section{Epidemiological characteristics}

During the study period, epidemiological and clinical data were collected on 101 medical staff with laboratory-confirmed SARS-Cov-2 infection from Wuhan Union Hospital, of whom 99 (98\%) provided an exact date of symptom onset and only 6 cases $(6 \%)$ were severe. The patients aged between 23 and 63 years old, and median age was 33 years (IQR 30-41 years) (Table 1). More than half of the cohort were female $(68 \%)$ and nurse $(74 \%)$. There were $18(18 \%)$ cases with a large BMI (BMI $\left.\geq 24 \mathrm{~kg} / \mathrm{m}^{2}\right)$, and $4(4 \%)$ were current smokers.

Among the 101 medical staff recruited, no one had an exposure to Huanan seafood wholesale market or wildlife, while $6(6 \%)$ medical staff had family members confirmed with SARS-CoV-2 infection. During patient care, $15(15 \%), 3(3 \%)$ and $3(3 \%)$ cases had direct contact with patients in fever clinics, isolation wards and specimens collected from patients, respectively. 10 (10\%) of 101 medical staff have used preventive medications. No major differences of exposure history and preventive medications were observed between the two occupational types, except for the contact with specimens were more common in doctors $(P$-value $<0.05)$.

In terms of entire cohort, the median time of onset to admission was 8.0 (IQR 5.0-15.0). There were similar probability density distributions for onset-todiagnosis, onset-to-admission, and diagnosis-toadmission intervals for nurse and doctor patients (Figure 1, A-C).

\section{Clinical features}

There were $19(19 \%)$ cases with one or more co-morbidities. The three most common symptoms were fever (70\%), cough (58\%) and fatigue $(39 \%)$. The relatively fewer common symptoms were sore throat, myalgia, difficulty breathing or chest tightness, sputum production, headache, chill, loss of appetite, diarrhea, and chest pain (Table 1). 
Table 1. Demographics and baseline characteristics of medical staff infected with COVID-19 pneumonia in Wuhan, China.

\begin{tabular}{|c|c|c|c|c|}
\hline & \multirow[t]{2}{*}{ All $(n=101)$} & \multicolumn{2}{|l|}{ Occupation } & \multirow[t]{2}{*}{$P$-value } \\
\hline & & Doctor $(n=26)$ & Nurse $(n=75)$ & \\
\hline Age, median (IQR) & $33(30-41)$ & $37(31-43)$ & $32(30-40)$ & 0.124 \\
\hline Sex & & & & $<0.001$ \\
\hline Male & $32(32 \%)$ & $18(69 \%)$ & $14(19 \%)$ & - \\
\hline Female & $69(68 \%)$ & $8(31 \%)$ & $61(71 \%)$ & - \\
\hline BMI $\left(\mathrm{kg} / \mathrm{m}^{2}\right)$ & $22.0(20.1-23.4)$ & $22.4(21.4-24.2)$ & $21.5(20.0-23.0)$ & 0.027 \\
\hline$\geq 24$ & $18(18 \%)$ & $8(31 \%)$ & $10(13 \%)$ & 0.071 \\
\hline$<24$ & $83(82 \%)$ & $18(69 \%)$ & $65(87 \%)$ & - \\
\hline Current smoking status & & & & 0.273 \\
\hline Yes & $4(4 \%)$ & $2(8 \%)$ & $2(3 \%)$ & - \\
\hline No & $97(96 \%)$ & $24(92 \%)$ & $73(97 \%)$ & - \\
\hline Disease severity & & & & 0.234 \\
\hline Non-severe & $95(94 \%)$ & $23(88 \%)$ & $72(96 \%)$ & - \\
\hline Severe & $6(6 \%)$ & $3(12 \%)$ & $3(4 \%)$ & - \\
\hline \multicolumn{5}{|l|}{ Exposure history } \\
\hline Exposure to Huanan market & 0 & 0 & 0 & - \\
\hline Exposure to wildlife & 0 & 0 & 0 & - \\
\hline Family members as confirmed cases & $6(6 \%)$ & $2(8 \%)$ & $4(5 \%)$ & 0.646 \\
\hline Contact with patients in fever clinics & $15(15 \%)$ & $3(12 \%)$ & $12(16 \%)$ & 0.754 \\
\hline Contact with patients in isolation wards & $3(3 \%)$ & $1(4 \%)$ & $2(3 \%)$ & 1 \\
\hline Contact with specimens & $3(3 \%)$ & $3(12 \%)$ & 0 & 0.016 \\
\hline Use of preventive medication & & & & 0.276 \\
\hline Yes & $10(10 \%)$ & $4(15 \%)$ & $6(8 \%)$ & - \\
\hline No & $91(90 \%)$ & $23(85 \%)$ & $69(92 \%)$ & - \\
\hline Comorbidities & $19(19 \%)$ & $4(15 \%)$ & $15(20 \%)$ & 0.774 \\
\hline Hypertension & $4(4 \%)$ & $1(4 \%)$ & $3(4 \%)$ & 1 \\
\hline Diabetes & $1(1 \%)$ & 0 & $1(1 \%)$ & 1 \\
\hline Coronary heart disease & $1(1 \%)$ & 0 & $1(1 \%)$ & 1 \\
\hline Chronic obstructive pulmonary disease & 0 & 0 & 0 & - \\
\hline Other & $14(14 \%)$ & $3(12 \%)$ & $11(15 \%)$ & 1 \\
\hline \multicolumn{5}{|l|}{ Signs and symptoms } \\
\hline Fever & $71(70 \%)$ & $16(62 \%)$ & $55(73 \%)$ & 0.376 \\
\hline Maximum temperature, ${ }^{\circ} \mathrm{C}$ & & & & 0.646 \\
\hline$\leq 37.3$ & $30(30 \%)$ & $10(38 \%)$ & $20(27 \%)$ & - \\
\hline 37.3-38 & $42(42 \%)$ & $10(38 \%)$ & $32(43 \%)$ & - \\
\hline $38-39$ & $20(20 \%)$ & $4(15 \%)$ & $16(21 \%)$ & - \\
\hline$>39$ & $6(6 \%)$ & $2(8 \%)$ & $4(5 \%)$ & - \\
\hline Cough & $59(58 \%)$ & $15(58 \%)$ & $45(59 \%)$ & 1 \\
\hline Fatigue & $39(39 \%)$ & $9(35 \%)$ & $30(40 \%)$ & 0.801 \\
\hline Sore throat & $24(24 \%)$ & $7(27 \%)$ & $17(23 \%)$ & 0.863 \\
\hline Myalgia & $18(18 \%)$ & $8(31 \%)$ & $10(13 \%)$ & 0.071 \\
\hline Difficulty breathing or chest tightness & $21(21 \%)$ & $3(12 \%)$ & $18(24 \%)$ & 0.285 \\
\hline Sputum production & $21(21 \%)$ & $4(15 \%)$ & $17(23 \%)$ & 0.611 \\
\hline Headache & $14(14 \%)$ & $4(15 \%)$ & $10(13 \%)$ & 0.752 \\
\hline Chill & $8(8 \%)$ & $2(8 \%)$ & $6(8 \%)$ & 1 \\
\hline Loss of appetite & $8(8 \%)$ & $2(8 \%)$ & $6(8 \%)$ & 1 \\
\hline Diarrhea & $11(11 \%)$ & $4(15 \%)$ & $7(9 \%)$ & 0.467 \\
\hline Chest pain & $5(5 \%)$ & $1(4 \%)$ & $4(5 \%)$ & 1 \\
\hline Time from symptoms onset to admission, median (IQR) (days) & $8.0(5.0-15.0)$ & $7.0(5.0-12.5)$ & $8.0(5.0-15.0)$ & 0.720 \\
\hline
\end{tabular}

Table 2 shows the laboratory and radiographic findings of 101 medical staff with confirmed COVID-19. On admission, the blood counts of 23 $(23 \%)$ cases showed leukocytopenia and only one (1\%) showed leukocytosis. $33(33 \%)$ presented with lymphocytopenia and $12(12 \%)$ presented with thrombocytopenia. Most cases demonstrated normal levels of D-dimer, creatinine, and creatine kinase, but elevated C-reactive protein and amyloid A levels were presented in $41 \%$ and $59 \%$ of cases, respectively.
Elevated levels of alanine aminotransferase (15\%) and aspartate aminotransferase $(8 \%)$ were less common. A small proportion $(2 \%)$ of cases had abnormal procalcitonin serum level (>0.5 ug/L). Notably, 80\% of cases had increased levels of IL-6 $(>2.9 \mathrm{pg} / \mathrm{ml})$. In contrast to nurses, doctors showed significantly higher levels of creatinine, creatine kinase, lactose dehydrogenase, alanine aminotransferase, aspartate aminotransferase, hemoglobin, ferritin, and total bilirubin (all $P$-values $<0.05$ ). 
(A)

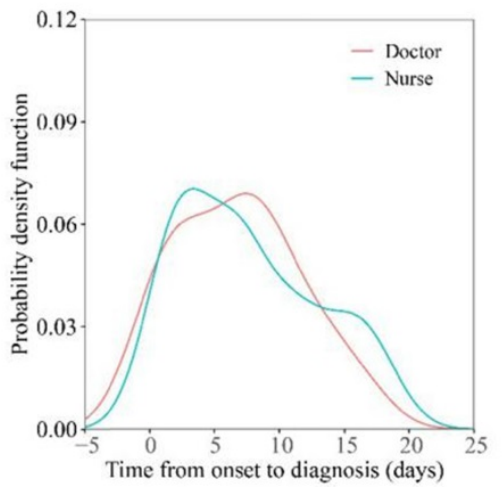

(B)

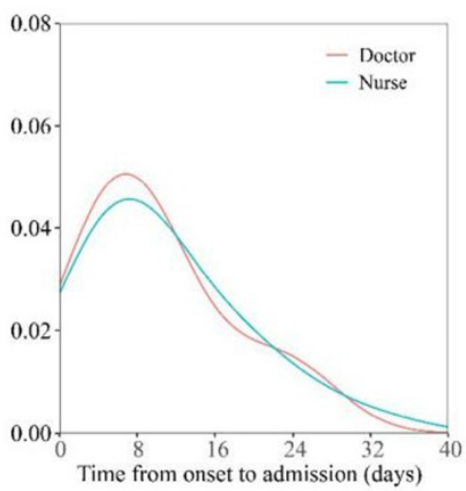

(C)

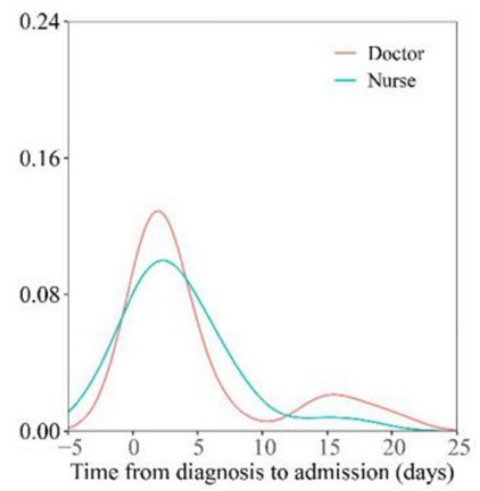

Figure 1. Distributions of date of diagnosis, onset-to-diagnosis duration, onset-to-admission duration and diagnosis-to-admission duration. (A) Estimates of onset-to-diagnosis distribution stratified by occupation. (B) Estimates of onset-to-admission distribution stratified by occupation. (C) Estimates of diagnosis-to-admission distribution stratified by occupation.

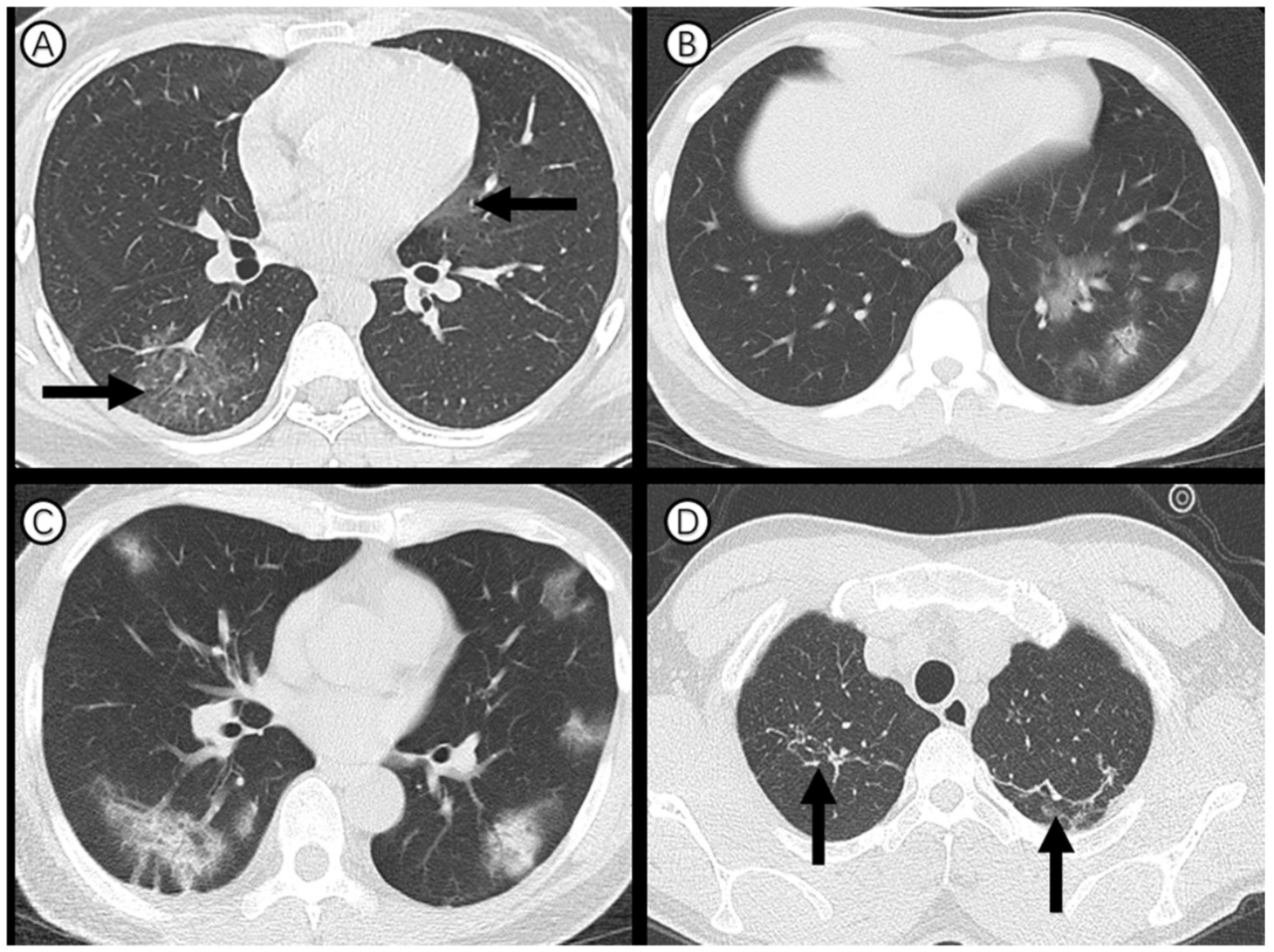

Figure 2. Axial thin-section CT scans in medical staff infected with SARS-CoV-2. (A) 27-year-old woman: bilateral, peripheral ground-glass opacity in the right lower lobe and left upper lobe (arrow). (B) 27-year-old man: unilateral, multiple and perbronchovasular ground-glass opacity associated with air bronchograms in the left lower lobe. (C) 55-year-old man: bilateral, peripheral ground-glass opacity mixed consolidation pattern. (D) 28-year-old man: bilateral and linear atelectasis in the right and left upper lobes (arrow), regarded as chronic lung lesion with lack of changes on serial CT examinations.

Ninety-one (90\%) of 101 cases showed abnormal chest CT (Figure 2). Sixty-three (62\%) had bilateral lung involvement (Figure 2, A and C). The right lower lobe $(68 \%)$ and left lower lobe $(70 \%)$ were the most common involved lobes. Ground glass opacity was the predominant abnormality on chest CT (Figure 2, A and B) and observed in 48 cases (48\%). Subpleural distribution was predominant distribution pattern of the ground glass opacity as identified in 78 cases $(77 \%)$ (Figure 2, C). Adjacent pleura thickening, nodules, emphysema, pleural effusion and lymphadenopathy were relatively rare. CT scans also found that $23(23 \%)$ of medical staff had one or more chronic lung lesions with unchanged appearance on serial CT examinations (Figure 2, D). 
Table 2. Laboratory and radiographic findings of medical staff infected with COVID-19 pneumonia in Wuhan, China.

\begin{tabular}{|c|c|c|c|c|c|}
\hline & \multirow[t]{2}{*}{ Normal range } & \multirow[t]{2}{*}{ All $(n=101)$} & \multicolumn{2}{|l|}{ Occupation } & \multirow[t]{2}{*}{$P$-value } \\
\hline & & & Doctor $(n=26)$ & Nurse $(n=75)$ & \\
\hline Leukocytes $\left(\times 10^{9} / \mathrm{L}\right)$ & $3.5-9.5$ & $4.5(3.6-5.7)$ & $4.8(3.7-5.6)$ & $4.4(3.5-5.7)$ & 0.545 \\
\hline Decreased & & $23(23 \%)$ & $4(15 \%)$ & $19(24 \%)$ & 0.441 \\
\hline Increased & & $1(1 \%)$ & 0 & $1(1 \%)$ & 1 \\
\hline Neutrophilic granulocyte percentage (\%) & $40-75$ & $56.1(49.9-62.7)$ & $59.5(52.0-65.1)$ & $54.4(49.1-62.4)$ & 0.162 \\
\hline Lymphocytes $\left(\times 10^{9} / \mathrm{L}\right)$ & $1.1-3.2$ & $1.4(0.9-1.8)$ & $1.3(0.9-1.9)$ & $1.4(1.0-1.7)$ & 0.706 \\
\hline Decreased & & $33(33 \%)$ & $12(46 \%)$ & $21(28 \%)$ & 0.145 \\
\hline Platelets $\left(\times 10^{9} / \mathrm{L}\right)$ & $115-350$ & $190(144-220)$ & $189(141-213)$ & $190(146-228)$ & 0.843 \\
\hline Decreased & & $12(12 \%)$ & $2(8 \%)$ & $10(13 \%)$ & 0.679 \\
\hline D-dimer $(\mathrm{mg} / \mathrm{L})^{*}$ & $0-0.5$ & $0.2(0.2,0.4)$ & $0.3(0.2,0.5)$ & $0.2(0.2,0.4)$ & 0.226 \\
\hline Creatinine (umol/L) & 44-106 & $65.8(58.6-77.2)$ & $76.3(65.4-87.5)$ & $62.6(57.9-70.7)$ & $<0.001$ \\
\hline Creatine kinase (U/L) & $26-140$ & $54.0(42.0-88.0)$ & $95.5(63.8-118)$ & $49.0(38.0-65.0)$ & $<0.001$ \\
\hline Lactose dehydrogenase (U/L) & $109-245$ & $187(167-217)$ & $214(177-274)$ & $185(164-204)$ & 0.024 \\
\hline Alanine aminotransferase $(\mathrm{U} / \mathrm{L})$ & $5-35$ & $19.0(13.0-29.0)$ & $26.5(19.3-49.8)$ & $16.0(13.0-26.5)$ & $<0.001$ \\
\hline Increased & & $15(15 \%)$ & $8(31 \%)$ & $7(9 \%)$ & 0.020 \\
\hline Aspartate aminotransferase (U/L) & $8-40$ & $22.0(16.0-26.0)$ & $24.5(22.0-32.5)$ & $20.0(16.0-25.0)$ & 0.002 \\
\hline Increased & & $8(8 \%)$ & $6(22 \%)$ & $2(3 \%)$ & 0.004 \\
\hline Hemoglobin (g/L) & $115-150$ & $128(119-139)$ & $139(128-150)$ & $126(119-134)$ & 0.002 \\
\hline Ferritin (ug/L) & $4.6-204$ & $99(55-247)$ & $329(206-553)$ & $82(43-155)$ & $<0.001$ \\
\hline C-reactive protein $>8 \mathrm{mg} / \mathrm{L}$ & $0-8$ & $41(41 \%)$ & $13(50 \%)$ & $28(37 \%)$ & 0.367 \\
\hline Amyloid A (mg/L) & $0-10$ & $22.4(5.0-129.4)$ & $39.6(7.5-334.3)$ & $15.5(4.8-78.6)$ & 0.067 \\
\hline Increased & & $50 / 85(59 \%)$ & $18 / 25(72 \%)$ & $32 / 60(53 \%)$ & 0.177 \\
\hline Procalcitonin $>0.5 \mathrm{ug} / \mathrm{L}$ & $0-0.5$ & $2 / 95(2 \%)$ & $1 / 23(4 \%)$ & $1 / 72(1 \%)$ & 0.428 \\
\hline Total bilirubin (umol/L) & $5.1-19$ & $8.9(7.5-12.5)$ & $11.1(8.7-14.7)$ & $8.5(67.0-13.4)$ & 0.003 \\
\hline IL-6 (pg/ml) & $0.1-2.9$ & $4.3(3.2-6.9)$ & $4.3(3.3-6.4)$ & $4.3(3.2-7.0)$ & 0.967 \\
\hline Increased & & $76 / 95(80 \%)$ & $21 / 25(84 \%)$ & $55 / 70(79 \%)$ & 0.771 \\
\hline \multicolumn{6}{|l|}{ lymphocyte subsets } \\
\hline $\mathrm{CD} 3+$ ratio $(\%)$ & $58.17-84.22$ & $75.2(70.1-79.9)$ & $70.5(64.0-76.9)$ & $77.3(71.9-80.6)$ & 0.019 \\
\hline CD4+ ratio (\%) & $25.34-51.37$ & $41.3(35.2-46.0)$ & $38.2(34.2-44.6)$ & $41.7(36.4-48.4)$ & 0.303 \\
\hline CD8+ ratio (\%) & 14.23-38.95 & $27.9(23.6-32.6)$ & $25.7(23.6-30.0)$ & $28.4(24.5-33.4)$ & 0.178 \\
\hline B-CELL ratio (\%) & 4.1-18.31 & $11.2(8.9-14.9)$ & $10.4(9.2-14.6)$ & $11.3(8.8-15.3)$ & 0.575 \\
\hline NK cell ratio (\%) & $3.33-30.47$ & $6.2(4.0-10.2)$ & $11.1(6.1-17.7)$ & $5.2(3.5-7.6)$ & 0.002 \\
\hline Ratio of CD4/CD8 & $0.41-2.72$ & $1.4(1.2-1.9)$ & $1.5(1.3-1.9)$ & $1.4(1.2-1.9)$ & 0.617 \\
\hline Abnormalities on chest $\mathrm{CT}$ & - & $91(90 \%)$ & $25(96 \%)$ & $66(88 \%)$ & 0.446 \\
\hline \multicolumn{6}{|l|}{ Lung involvement } \\
\hline Unilateral & - & $28(28 \%)$ & $8(31 \%)$ & $20(27 \%)$ & 0.882 \\
\hline Bilateral & - & $63(62 \%)$ & $17(65 \%)$ & $46(61 \%)$ & 0.895 \\
\hline \multicolumn{6}{|l|}{ Lung lobe involved } \\
\hline Right upper lobe & - & $35(35 \%)$ & $12(46 \%)$ & $23(31 \%)$ & 0.234 \\
\hline Right middle lobe & - & $29(29 \%)$ & $15(58 \%)$ & $14(19 \%)$ & $<0.001$ \\
\hline Right lower lobe & - & $69(68 \%)$ & $20(77 \%)$ & $49(65 \%)$ & 0.395 \\
\hline Left upper lobe & - & $39(39 \%)$ & $16(62 \%)$ & $23(31 \%)$ & 0.011 \\
\hline Left lower lobe & - & $71(70 \%)$ & $17(65 \%)$ & $54(72 \%)$ & 0.699 \\
\hline \multicolumn{6}{|l|}{ Predominant CT pattern } \\
\hline Ground glass opacity & - & $48(48 \%)$ & $12(46 \%)$ & $36(48 \%)$ & 1 \\
\hline Consolidation & - & $13(13 \%)$ & $4(15 \%)$ & $9(12 \%)$ & 0.534 \\
\hline Mixed pattern & - & $30(30 \%)$ & $9(35 \%)$ & $21(28 \%)$ & 0.699 \\
\hline \multicolumn{6}{|l|}{ Predominant distribution of opacities } \\
\hline Septal/subpleural & - & $78(77 \%)$ & $21(81 \%)$ & $57(76 \%)$ & 0.819 \\
\hline Peribronchovascular & - & $4(4 \%)$ & 0 & $4(5 \%)$ & 0.570 \\
\hline Random & - & $9(9 \%)$ & $4(15 \%)$ & $5(7 \%)$ & 0.230 \\
\hline Thickening of the adjacent pleura & - & $4(4 \%)$ & $2(8 \%)$ & $2(3 \%)$ & 0.272 \\
\hline Pleural effusion/ Lymphadenopathy & & 0 & 0 & 0 & - \\
\hline Any original chronic lung lesion & - & $23(23 \%)$ & $7(27 \%)$ & $16(21 \%)$ & 0.753 \\
\hline
\end{tabular}

Data are presented as medians (interquartile ranges, IQR) and $n$ (\%). For each item, the effective sample size of total population, group of doctors, group of nurses is 101,26 and 75 .

\section{Treatment measures and prognosis}

Of the study subjects, no person was transferred to an intensive care unit for mechanical ventilation due to acute respiratory distress syndrome. Empirical intravenous antibiotic treatment was administered in $84(83 \%)$ patients. All the patients were given empirical antiviral therapy. Meanwhile, 37 (37\%) were offered traditional Chinese medicine, 34 (34\%) patients were given immune globulin, 58 (57\%) were given thymosin, and $11 \quad(11 \%)$ received corticosteroids. As for oxygen therapy, 56 (55\%) used nasal cannula and only $7(7 \%)$ used face mask, while no one needed invasive mechanical or ventilation extracorporeal membrane oxygenation. 


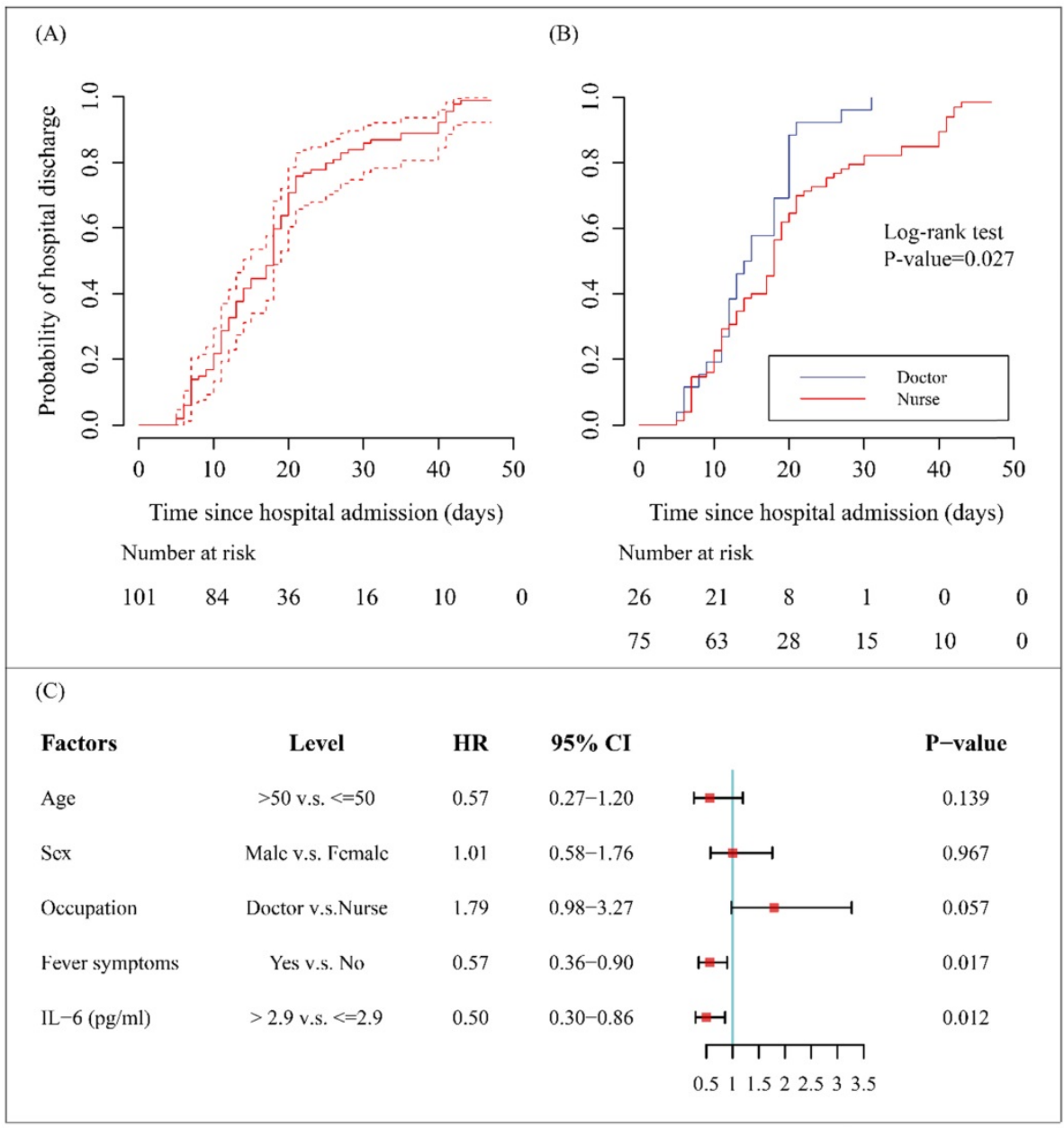

Figure 3. Hospital discharge rates and factors associated with outcome for medical staff infected with COVID-19 pneumonia. (A) The probability of hospital discharge and the length of hospitalization for the study subjects. Dotted arrows represent $95 \% \mathrm{Cls}$. (B) The probability of hospital discharge and the length of hospitalization stratified by occupation (doctor or nurse). (C) The results of proportional hazard Cox model. Hazard ratios (HRs) and corresponding $95 \%$ Cls were displayed for the factors including age, sex, occupation, fever symptoms and IL-6 levels.

Table 3. Treatments and outcomes of medical staff infected with COVID-19 pneumonia in Wuhan, China.

\begin{tabular}{|c|c|c|c|c|}
\hline & \multirow[t]{2}{*}{ All $(n=101)$} & \multicolumn{2}{|l|}{ Occupation } & \multirow[t]{2}{*}{$P$-value } \\
\hline & & Doctor $(n=26)$ & Nurse $(n=75)$ & \\
\hline $\begin{array}{l}\text { Electrocardiograph } \\
\text { monitoring }\end{array}$ & $24(24 \%)$ & $6(23 \%)$ & $18(24 \%)$ & 1 \\
\hline Antibiotics treatment & $84(83 \%)$ & $19(73 \%)$ & $65(87 \%)$ & 0.132 \\
\hline Antiviral treatment & $101(100 \%)$ & $26(100 \%)$ & $75(100 \%)$ & - \\
\hline $\begin{array}{l}\text { Traditional Chinese } \\
\text { medicine }\end{array}$ & $37(35 \%)$ & $7(26 \%)$ & $30(38 \%)$ & 0.401 \\
\hline Immune globulin & $34(34 \%)$ & $9(35 \%)$ & $25(33 \%)$ & 1 \\
\hline Thymosin & $58(57 \%)$ & $14(54 \%)$ & $44(59 \%)$ & 0.843 \\
\hline Corticosteroids & $11(11 \%)$ & $2(8 \%)$ & $9(12 \%)$ & 0.724 \\
\hline \multicolumn{5}{|l|}{ Oxygen therapy } \\
\hline Nasal cannula & $56(55 \%)$ & $13(50 \%)$ & $43(57 \%)$ & 0.675 \\
\hline Face mask & $7(7 \%)$ & $3(12 \%)$ & $4(5 \%)$ & 0.370 \\
\hline $\begin{array}{l}\text { Length of hospital stay } \\
\text { (days) }\end{array}$ & $\begin{array}{l}17.0 \\
(11.0-21.0)\end{array}$ & $14.0(11.3-20.0)$ & $18.0(11.0-23.0)$ & 0.232 \\
\hline Outcome & & & & 0.567 \\
\hline Hospital discharge & $98(97 \%)$ & $26(100 \%)$ & $72(96 \%)$ & - \\
\hline $\begin{array}{l}\text { Continued } \\
\text { hospitalization }\end{array}$ & $3(3 \%)$ & 0 & $3(4 \%)$ & - \\
\hline Death & 0 & 0 & 0 & - \\
\hline
\end{tabular}

By 20 Mar 2020, 98 (97\%) of the cases have been discharged and none had died, the remaining 3 cases were still in hospital to receive supportive therapy. According to the results of Kaplan-Meier method, the median discharge time (i.e. equal to the time that half of the patients left the hospital) of the entire cohort was 18.0 (95\% CI, 14.0-19.0) days (Figure 3, A). The accumulative probability of hospital discharge was higher in doctors versus nurses examined by log-rank test $(P$-value $<0.05)$ (Figure 3, B).

It should note that the endpoint of final multivariate Cox regression model was discharge, and patients who continued to be hospitalized as of 20 Mar 2020 were regarded as censored data. Fever symptoms (HR=0.57; 95\% CI 0.36-0.90) and elevated IL-6 levels $(>2.9 \mathrm{pg} / \mathrm{ml})$ on admission $(\mathrm{HR}=0.50 ; 95 \%$ CI 0.30-0.86) were unfavorable factors for discharge (all HRs $<1$ and all $P$-values <0.05) (Figure 3, C). However, the differences in discharge rates between the two occupational types tended to be marginally significant after adjusting for others covariates $(P$-value $=0.057)$ (Figure 3, C). 


\section{Discussion}

By 20 Feb, 2020 the China CDC Weekly reported a total of 2,055 laboratory-confirmed cases of medical staff with SARS-CoV-2 infection, of which the majority (88\%) were from Hubei province [19]. The exact mode of the most medical staff infection (73\%) remains unclear in our study, in consistency with the findings reported by Wei X-S et al. [11] and Chu J et al. [13] respectively. Differ from some published studies about ordinary people [8, 9], we found that the infection of SARS-Cov-2 among medical staff mainly occurred at the early stage of COVID-19 epidemic in Wuhan. Possible reasons for these phenomena include lack of knowledge about transmission approaches and experience to fight with the SARS-CoV-2, coupled with a shortage of protection supplies at the early stage [15]. Therefore, training the health care professionals on protection techniques and standardized protection process, and providing adequate protective materials may play an important role in preventing infection of the medical staff and facilitating infection control.

The demographic characteristics and clinical manifestations of medical staff with confirmed COVID-19 in Wuhan were not exactly the same as general confirmed patients included in recent studies $[10,20,21]$. In our study, most of the novel coronavirus-infected medical staff analyzed were females and nurses, and were younger, in consistency with the findings reported by Xiong $W$ et al [12]. The medical staff infected with SARS-CoV-2 have similar signs and symptoms with general confirmed infection patients $[10,21]$. The infected medical staff tended to have bilateral, subpleural ground-glass opacities on chest CT images, which is consistent with the recent radiological reports on COVID-19 pneumonia [22-26]. Furthermore, abnormal D-dimer levels as well as abnormal renal, heart and liver function tests were relatively rare among medical staff with SARS-CoV-2 infection.

In our study, only 6 of the 101 medical staffs with SARS-CoV-2 infection were severe case. None developed acute respiratory distress syndrome or transferred to intensive care unit. The low rate of severe and critical case $(5.9 \%, 6 / 101)$ of the medical staff infections in our study is similar to the rate of severe cases $(5 \%, 4 / 80)$ reported by Wuhan Tongji Hospital, which is a different hospital affiliated to the same university as ours. Previous studies suggested that $13.8 \%$ of the general confirmed patients were severe cases, among whom older age, male sex, chronic diseases are more common [27-29]. In our study, the medical staffs have relatively milder symptoms, which may be partly due to their medical expertise, younger age and less underlying diseases.
Predictors of hospital discharge among infected medical staff were identified by Cox model. Fever symptoms and elevated IL-6 levels $(>2.9 \mathrm{pg} / \mathrm{ml})$ on admission were significantly associated with lower likelihood of the discharge. Knowledge of how present-on-admission patient factors affect patient's condition and risk during hospitalization is very important because we can use such knowledge to screen and identify patients of higher risk upon admission to the hospital. A recent study revealed that fever was identified in only half of the patients on presentation but increased to nearly $90 \%$ after hospitalization [21]. Elevated IL-6 levels were observed in $80 \%$ of infected medical staff on admission, which is associated with inflammatory response [30, 31]. The elevated inflammatory cytokines (such as IL-6 and IL-1) suggest that a cytokine storm may play a major role in the pathology of COVID-19 [32].

So far, more than 40,000 health-care workers from 30 provinces gathered in Wuhan for the battle against the epidemic. China has attached great importance to infection prevention among medical staff including providing adequate protective materials (gown, gloves, N95 respirator, face shield or goggles) and training the health care standardized protection process, and the good news was that none of them were infected with SARS-CoV-2 up to now. Meanwhile, some potential problems remain to be solved, such as unclear patterns of infection, mental health care for medical staff [33], and the possibility of airborne transmission from aerosol production by medical practices in health care facilities. A recent study from Singapore found that surface environmental and personal protective equipment contamination caused by respiratory droplets and fecal shedding from patients infected with SARS-CoV-2, suggesting that the environment is a potential viral vector [34]. Further investigations should be devoted to identifying the exact patterns of SARS-CoV-2 infection among medical staff.

\section{Limitations of this study}

We acknowledge some limitations of this study. First, only 101 medical staff from a single hospital in Wuhan were included in this study. This limitation may result in deviations in epidemiological and clinical observation characteristics, particularly regarding specific causes of SARS-CoV-2 infection among medical staff; Second, this is a retrospective study and the data used in this study only provide a preliminary insight into epidemiological features and clinical outcomes of a group of medical staff confirmed with COVID-19. Further research on this regard is needed. 


\section{Conclusion}

The infection among medical staff mainly occurring at the early stages of COVID-19 epidemic in Wuhan was suggested. In this study, medical staffs infected with COVID-19 have relatively milder symptoms and favorable clinical course than other ordinary patients, which may be partly due to their medical expertise, younger age and less underlying diseases. The major differences between the two occupational types manifested in laboratory indicators. The potential risk factors of presence of fever symptoms and IL-6 levels greater than >2.9 $\mathrm{pg} / \mathrm{ml}$ could help to identify medical staff with poor prognosis at an early stage. Further investigations should be devoted to identifying the exact mode of COVID-19 among medical staff.

\section{Acknowledgments}

We would like to thank all colleagues for helping us during the current study. The authors would like to express their appreciation for all the emergency services, nurses, doctors, and other hospital staff for their efforts to combat the COVID-19 outbreak. We also would like to thank Prof. Pi Guo (Department of Preventive Medicine, Shantou University Medical College, Shantou, Guangdong, China) for statistical supporting.

\section{Funding statement}

This study was funded by the grant from the COVID-19 Rapid Response Call of Huazhong University of Science and Technology (No.2020kfyXGYJ021) and the grant from the Hubei Province Key Laboratory of Molecular Imaging (No.2020fzyx009). The funder of the study had no role in study design, data collection, data analysis, data interpretation, or writing of the report.

\section{Author contributions statement}

J.L., L.OY. and C.S.Z. conceptualized the article. J.L., L.OY., D.Y., X.Y.H. and Y.K.C. collected the epidemiological, clinical and radiological data. J.L. and L.OY. cleaned and analyzed the data. J.L. and F.Y. contributed to radiological data interpretation. J.L. and L.OY. drafted the manuscript. O.A., H.P.W., H.S.S., F.Y., and C.S.Z. revised the final manuscript. All authors approved the final draft of the manuscript. C.S.Z. is the guarantor. The corresponding author attests that all listed authors meet authorship.

\section{Competing Interests}

The authors have declared that no competing interest exists.

\section{References}

1. Huang C, Wang Y, Li X, Ren L, Zhao J, Hu Y, Zhang L, Fan G, Xu J, Gu X, et al. Clinical features of patients infected with 2019 novel coronavirus in Wuhan, China. The Lancet. 2020; 395(10223):497-506.

2. Lu R, Zhao X, Li J, Niu P, Yang B, Wu H, Wang W, Song H, Huang B, Zhu N, et al. Genomic characterisation and epidemiology of 2019 novel coronavirus: implications for virus origins and receptor binding. The Lancet. 2020; 395(10224):565-574.

3. Zhu N, Zhang D, Wang W, Li X, Yang B, Song J, Zhao X, Huang B, Shi W, Lu R, et al. A Novel Coronavirus from Patients with Pneumonia in China, 2019. New England Journal of Medicine. 2020; 382(8):727-733.

4. [Internet] World Health Organization. Coronavirus Disease 2019 (COVID-19) Situation Reports. https://www.who.int/emergencies/diseases/novelcoronavirus-2019/situation-reports. Published 5 October, 2020. Accessed 5 October, 2020.

5. Rothe C, Schunk M, Sothmann P, Bretzel G, Froeschl G, Wallrauch C, Zimmer T, Thiel V, Janke C, Guggemos W, et al. Transmission of 2019-nCoV Infection from an Asymptomatic Contact in Germany. New England Journal of Medicine 2020, 382(10):970-971.

6. Li Q, Guan X, Wu P, Wang X, Zhou L, Tong Y, Ren R, Leung K, Lau E, Wong JY, et al. Early Transmission Dynamics in Wuhan, China, of Novel Coronavirus-Infected Pneumonia. New England Journal of Medicine. 2020; 382:1199-1207.

7. Wu JT, Leung K, Leung GM. Nowcasting and forecasting the potential domestic and international spread of the 2019-nCoV outbreak originating in Wuhan, China: a modelling study. The Lancet. 2020; 395(10225):689-697.

8. Chen N, Zhou M, Dong X, Qu J, Gong F, Han Y, Qiu Y, Wang J, Liu Y, Wei Y, et al. Epidemiological and clinical characteristics of 99 cases of 2019 novel coronavirus pneumonia in Wuhan, China: a descriptive study. The Lancet. 2020; 395(10223):507-513.

9. Wang D, Hu B, Hu C, Zhu F, Liu X, Zhang J, Wang B, Xiang H, Cheng Z, Xiong Y, et al. Clinical Characteristics of 138 Hospitalized Patients With 2019 Novel Coronavirus-Infected Pneumonia in Wuhan, China. JAMA. 2020; 323(11):1061-1069.

10. The Novel Coronavirus Pneumonia Emergency Response Epidemiology T: The Epidemiological Characteristics of an Outbreak of 2019 Novel Coronavirus Diseases (COVID-19) - China, 2020. China CDC Weekly 2020, 2(8):113-122.

11. Wei X-S, Wang X-R, Zhang J-C, Yang W-B, Ma W-L, Yang B-H, Jiang N-C, Gao Z-C, Shi H-Z, Zhou Q. A cluster of health care workers with COVID-19 pneumonia caused by SARS-CoV-2. Journal of Microbiology, Immunology and Infection. 2020.

12. Wang X, Liu W, Zhao J, Lu Y, Wang X, Yu C, Hu S, Shen N, Liu W, Sun Z, et al. Clinical characteristics of 80 hospitalized frontline medical workers infected with COVID-19 in Wuhan, China. Journal of Hospital Infection. 2020; 105(3):399-403.

13. Chu J, Yang N, Wei Y, Yue H, Zhang F, Zhao J, He L, Sheng G, Chen P, Li G, et al. Clinical characteristics of 54 medical staff with COVID-19: A retrospective study in a single center in Wuhan, China. Journal of Medical Virology. 2020; 92(7):807-813.

14. The L: COVID-19: protecting health-care workers. The Lancet. 2020; 395(10228):922.

15. Xiang Y-T, Jin Y, Wang Y, Zhang Q, Zhang L, Cheung T: Tribute to health workers in China: A group of respectable population during the outbreak of the COVID-19. International Journal of Biological Sciences. 2020; 16(10):1739-1740.

16. [Internet] WHO. Coronavirus disease (COVID-19) technical guidance: laboratory testing for 2019-nCoV in humans. https://www.who.int/ emergencies/diseases/novel-coronavirus-2019/technical-guidance/laborator y-guidance (accessed Feb 22, 2020).

17. [Internet] The International Severe Acute Respiratory and Emerging Infection Consortium (ISARIC). https://isaric.tghn.org/ (accessed Feb 23, 2020).

18. [Internet] National Health Commission of the People's Republic of China. New coronavirus pneumonia diagnosis and treatment program (trial version 5, revised version). http://www.nhc.gov.cn/yzygj/s7652m/202002/ 41c3142b38b84ec4a748e60773cf9d4f.shtml (assessed Feb 23, 2020).

19. [Internet] Central People's Government of the People's Republic of China. China-WHO joint investigation report for novel coronavirus pneumonia (COVID-19). Feb 29, 2020. http://www.nhc.gov.cn/jkj/s3578/202002/ 87fd92510d094e4b9bad597608f5cc2c.shtml (assessed Mar 1, 2020).

20. Xu X-W, Wu X-X, Jiang X-G, Xu K-J, Ying L-J, Ma C-L, Li S-B, Wang H-Y, Zhang S, Gao H-N, et al. Clinical findings in a group of patients infected with the 2019 novel coronavirus (SARS-Cov-2) outside of Wuhan, China: retrospective case series. BMJ. 2020; 368:m606.

21. Guan W-J, Ni Z-y, Hu Y, Liang W, Ou C-Q, He J-x, Liu L, Shan H, Lei C-l, Hui D, et al. Clinical Characteristics of Coronavirus Disease 2019 in China. New England Journal of Medicine. 2020; 382:1708-1720.

22. Shi H, Han X, Jiang N, Cao Y, Alwalid O, Gu J, Fan Y, Zheng C. Radiological findings from 81 patients with COVID-19 pneumonia in Wuhan, China: a descriptive study. The Lancet Infectious Diseases. 2020; 20(4):425-434.

23. Kong W, Agarwal P. Chest Imaging Appearance of COVID-19 Infection. Radiology: Cardiothoracic Imaging. 2020; 2:e200028. 
24. Pan F, Ye T, Sun P, Gui S, Liang B, Li L, Zheng D, Wang J, Hesketh R, Yang L, et al. Time Course of Lung Changes On Chest CT During Recovery From 2019 Novel Coronavirus (COVID-19) Pneumonia. Radiology. 2020; 295:200370.

25. Liu H, Luo S, Li H, Zhang Y, Huang C, Li X, Tan Y, Chen M: Clinical characteristics and longitudinal chest $\mathrm{CT}$ features of healthcare workers hospitalized with coronavirus disease 2019 (COVID-19). International Journal of Medical Sciences. 2020; 17(17):2644-2652.

26. Dai M, Liu X, Zhu X, Liu T, Xu C, Ye F, Yang L, Zhang Y: Temporal changes of CT findings between non-severe and severe cases of COVID-19 pneumonia: a multi-center, retrospective, longitudinal Study. International Journal of Medical Sciences. 2020; 17(17):2653-2662.

27. Yang X, Yu Y, Xu J, Shu H, Xia Ja, Liu H, Wu Y, Zhang L, Yu Z, Fang M, et al. Clinical course and outcomes of critically ill patients with SARS-CoV-2 pneumonia in Wuhan, China: a single-centered, retrospective, observational study. The Lancet Respiratory Medicine. 2020; 8(5):475-481.

28. Liang W, Guan W, Chen R, Wang W, Li J, Xu K, Li C, Ai Q, Lu W, Liang H, et al. Cancer patients in SARS-CoV-2 infection: a nationwide analysis in China. The Lancet Oncology. 2020; 21(3):335-337.

29. Wu Z, McGoogan JM. Characteristics of and Important Lessons From the Coronavirus Disease 2019 (COVID-19) Outbreak in China: Summary of a Report of 72314 Cases From the Chinese Center for Disease Control and Prevention. JAMA. 2020; 323(13):1239-1242.

30. Tisoncik Go J, Korth M, Simmons C, Farrar J, Martin T, Katze M. Into the Eye of the Cytokine Storm. Microbiology and molecular biology reviews : MMBR. 2012; 76:16-32

31. Yasukawa H, Ohishi M, Mori H, Murakami M, Chinen T, Aki D, Hanada T, Takeda K, Akira S, Hoshijima M, et al. IL-6 induces an anti-inflammatory response in the absence of SOCS3 in macrophages. Nature Immunology. 2003; 4(6):551-556

32. McGonagle D, Sharif K, O'Regan A, Bridgewood C. The Role of Cytokines including Interleukin-6 in COVID-19 induced Pneumonia and Macrophage Activation Syndrome-Like Disease. Autoimmunity Reviews. 2020; 19(6):102537.

33. Chen $\mathrm{O}$, Liang M, Li Y, Guo J, Fei D, Wang L, He L, Sheng C, Cai Y, Li X, et al. Mental health care for medical staff in China during the COVID-19 outbreak. Lancet Psychiatry. 2020; 7(4e):15-16

34. Ong SWX, Tan YK, Chia PY, et al. Air, Surface Environmental, and Personal Protective Equipment Contamination by Severe Acute Respiratory Syndrome Coronavirus 2 (SARS-CoV-2) From a Symptomatic Patient. The Journal of the American Medical Association. 2020; 323(16):1610-1612. 